\title{
IFU Spectroscopy of BCGs and Their Companions: Angular Momentum and Dynamical Mass
}

\author{
Jimmy $^{* a}, K^{\dagger}$ Kim-Vy $\operatorname{Tran}^{a}$, Sarah Brough ${ }^{b}$, Karl Gebhardt $^{c}$, Anja von der Linden ${ }^{d, e, f}$, \\ Warrick J. Couch ${ }^{g}$, Rob Sharp ${ }^{h}$ \\ ${ }^{a}$ George P. and Cynthia W. Mitchell Institute for Fundamental Physics and Astronomy, \\ Department of Physics and Astronomy, Texas A\&M University, College Station, TX 77843, USA \\ ${ }^{b}$ Australian Astronomical Observatory, PO Box 915, North Ryde, NSW 1670, Australia \\ ${ }^{c}$ Department of Astronomy, University of Texas at Austin, 1 University Station C1400, Austin, TX \\ 78712, USA \\ ${ }^{d}$ Kavli Institute for Particle Astrophysics and Cosmology, Stanford University, 452 Lomita Mall, \\ Stanford, CA 94305-4085, USA \\ ${ }^{e}$ Department of Physics, Stanford University, 382 Via Pueblo Mall, Stanford, CA 94305-4060 \\ ${ }^{f}$ Dark Cosmology Centre, Niels Bohr Institute, University of Copenhagen, Juliane Maries Vej \\ 30, 2100 Copenhagen Ø, Denmark \\ ${ }^{g}$ Centre for Astrophysics and Supercomputing, Swinburne University, PO Box 218, Hawthorn, \\ VIC 3122, Australia \\ ${ }^{h}$ Research School of Astronomy \& Astrophysics, Australian National University, Cotter Road, \\ Weston Creek, ACT 2611, Australia
}

Using the VIMOS Integral Field Unit (IFU) spectrograph on the Very Large Telescope (VLT), we have spatially mapped the kinematic properties of 10 nearby Brightest Cluster Galaxies (BCGs) and 4 BCG companion galaxies located within a redshift of $z=0.1$. In the hierarchical formation model, these massive galaxies $\left(10^{10.5} M_{\odot}<M_{d y n}<10^{11.9} M_{\odot}\right)$ are expected to undergo more mergers than lower mass galaxies, and simulations show that dry minor mergers can remove angular momentum. We test whether BCGs have low angular momenta by using the $\lambda_{R e}$ parameter developed by the SAURON and ATLAS ${ }^{3 \mathrm{D}}$ teams and combine our kinematics with Sloan Digital Sky Survey (SDSS) photometry to analyze the BCGs' merger status. We find that 30\% (3/10) of the BCGs and $100 \%$ of the companion galaxies (4/4) are fast rotators as defined by the ATLAS ${ }^{3 \mathrm{D}}$ criteria. We find that above $M_{d y n} \sim 11.5 M_{\odot}$, virtually all galaxies regardless of environment are slow rotators. To search for signs of recent merging, we analyze the photometry of each system and use the $G-M_{20}$ selection criteria to identify mergers. We find that $40 \pm 20 \%$ of our BCGs are currently undergoing or have recently undergone a merger (within 0.2 Gyrs). Surprisingly, we find no correlation between galaxies with high angular momentum and morphological signatures of merging.

Frank N. Bash Symposium 2013: New Horizons in Astronomy (BASH 2013)

October 6-8, 2013

Austin, Texas

\footnotetext{
* Speaker.

${ }^{\dagger}$ E-mail: Jimmy@physics.tamu.edu
} 


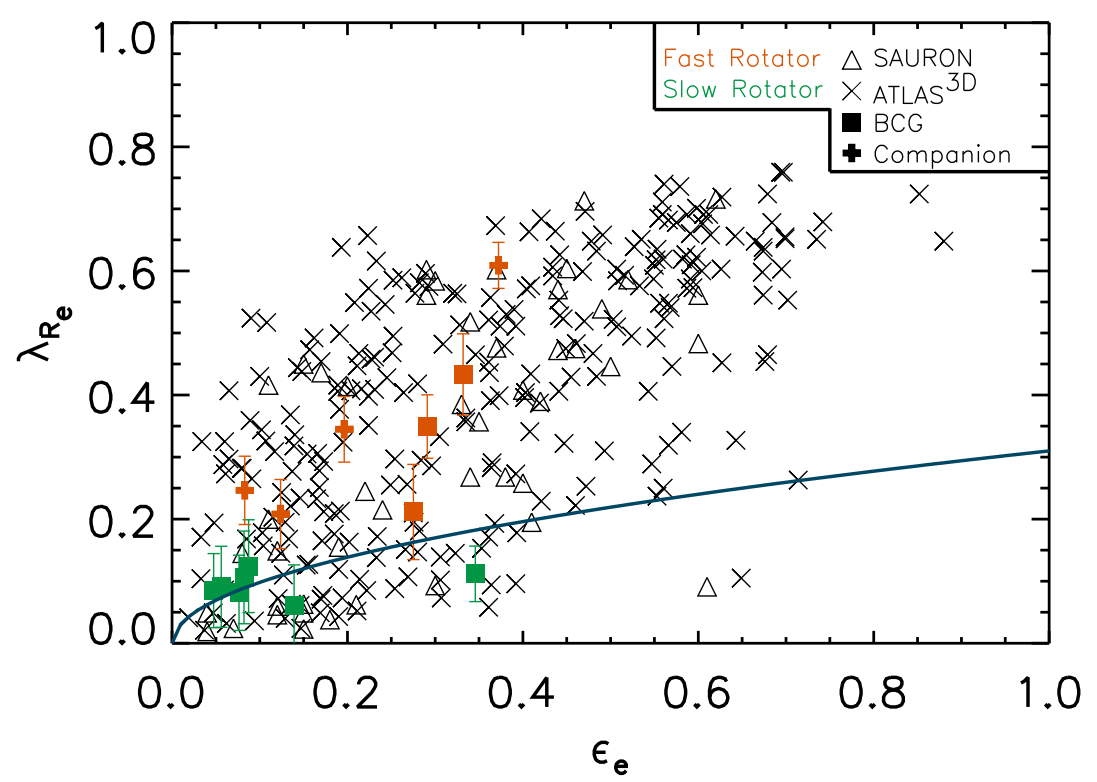

Figure 1: Lambda at the effective radius as a function of ellipticity, also measured at the effective radius. SAURON galaxies are plotted as triangles, ATLAS $^{3 \mathrm{D}}$ galaxies are plotted as crosses, BCGs from this study are plotted as squares, and Companion galaxies from this study are plotted as plus symbols. The blue line indicates the division between fast rotating and slow rotating galaxies. We find that three BCGs $(1048,1153$, $1261)$ and four companions $(1027,1066,1048)$ are classified as fast rotators.

Brightest Cluster Galaxies (BCGs) are expected to undergo more dry minor mergers than a typical elliptical galaxy, and dry minor mergers have been shown in simulations to decrease a galaxy's angular momentum ([1], [2], [3], [4]). As the result of many dry minor mergers, we do not expect BCGs to be fast rotating galaxies (as defined by ATLAS ${ }^{3 \mathrm{D}}$; [5]). We use our sample of 10 BCGs (selected from the SDSS-C4-DR3 catalogue [6] of BCGs with companions within 10") and their companions to search for a connection between angular momentum, dynamical mass, and very recent merger history.

Using observations from the VIMOS IFU spectrograph [7] on the VLT we calculate the $\lambda_{R}$ values that act as a proxy for angular momentum [8]. $\lambda_{R}$ is defined as

$$
\lambda_{R} \equiv \frac{\langle R|V|\rangle}{\left\langle R \sqrt{V^{2}+\sigma^{2}}\right\rangle}
$$

where $\mathrm{R}$ is the distance of the spaxel to the galaxy center, $\mathrm{V}$ is the velocity of the spaxel, and $\sigma$ is the velocity dispersion. The brackets in the numerator and the denominator denote a luminosity weighted average. We measure $\lambda_{R e}, \lambda_{R}$ at $1 R_{e}$ (effective radius), and find $\lambda_{R e}$ values ranging from $0.07 \pm 0.07$ to $0.60 \pm 0.04$ out of a possible range 0.0 to 1.0 (Figure 1). We have shown that $30 \%$ (3/10) of BCGs show signs of galaxy scale rotation and fit the ATLAS ${ }^{3 \mathrm{D}}$ definition of a fast rotating galaxy and the other $70 \%$ of our BCGs are slow rotating galaxies. We find the rather surprising result that $100 \%$ (4/4) of our companion galaxies are fast rotating.

We calculate $M_{d y n}$ for our sample of galaxies, finding dynamical masses up to $M_{d y n}=10^{11.9} M_{\odot}$. BCGs are some of the most massive galaxies in the universe, and the BCGs in our sample are among the most massive galaxies to have their angular momentum calculated using $\lambda_{R}$ (Figure 2). 

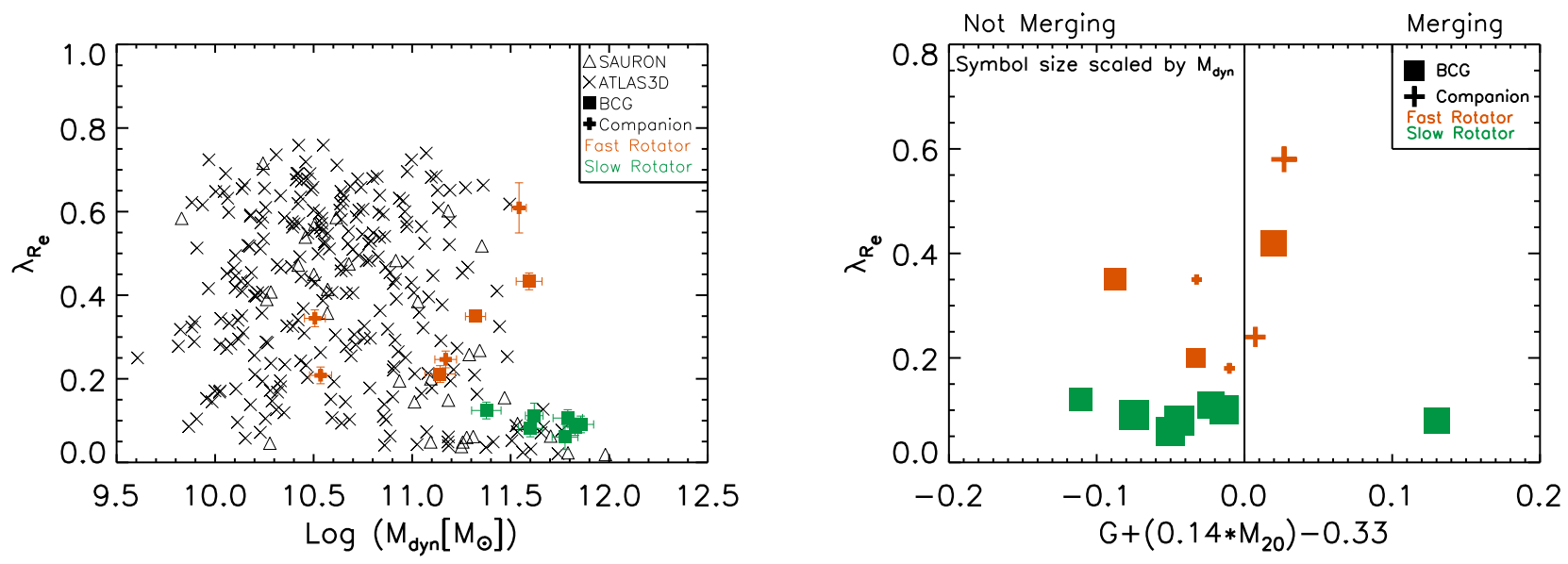

Figure 2: Left: Angular momentum parameter, $\lambda_{R e}$ versus dynamical mass, $M_{d y n}$. SAURON galaxies are plotted as triangles, ATLAS ${ }^{3 \mathrm{D}}$ galaxies are plotted as crosses, BCGs from this study are plotted as squares, and Companion galaxies from this study are plotted as plus symbols. The galaxies presented here are amongst the most massive studied. An upper mass limit to fast rotating galaxies can be seen in all samples around approximately $M_{d y n}=10^{11.5} M_{\odot}$. Right: Merger status and angular momentum at the effective radius. BCGs are plotted as squares, and companions are plotted as crosses. Plot symbols are scaled according to galaxy mass, with a larger symbol indicating a higher dynamical mass. Plot symbols are also color coded, with orange indicating a fast rotating galaxy, and green indicating a slow rotating galaxy. Galaxies plotted on the negative side of the x-axis are classified as not merging by the $G-M_{20}$ criteria, galaxies on the positive side of the $\mathrm{x}$-axis are classified as merging. There appears to be no correlation between Merging and $\lambda_{R e}$.

While we do find a small number of fast rotating BCGs (Figure 2), we find fewer fast rotating galaxies above $M_{d y n}=10^{11.5} M_{\odot}$, consistent with the previous limit for fast rotating galaxies in the SAURON and ATLAS ${ }^{3 \mathrm{D}}$ sample ([8], [9]). There appears to be an upper limit to how massive a galaxy can be while still maintaining a high angular momentum. The consistency between our results and that of the ATLAS ${ }^{3 \mathrm{D}}$ survey suggests that a galaxy's mass may be more significant than its position inside a cluster halo in determining angular momentum.

We examine the ongoing and recent merging history of the galaxies in our sample using the $G-M_{20}$ merger selection criteria [10]. We see that $40 \%$ (4/10) of systems in our sample are currently undergoing or have undergone a merger within the last 0.2 Gyrs (Figure 2). We use these merging results to examine the connection between $\lambda_{R e}$ and $G-M_{20}$. We find no correlation between $\lambda_{R e}$ and $G-M_{20}$. We find in 1 BCG that the minor merger event is likely responsible for the high $\lambda_{R e}$ value observed in the BCG and its companions. Conversely we find 2 BCGs that are fast rotating without any recent or ongoing merger events. In the slow rotating BCG category we find 3 mergers connected to a slow rotating BCG, and 4 examples of slow rotating BCGs without any mergers.

These diverse characteristics suggest that there is no strong connection between recent dry mergers and the $\lambda_{R e}$ classification as a fast or slow rotator. It is possible that complications from an unrelaxed dynamical system have skewed $\lambda_{R e}$ measurements in merging systems, or that the removal of angular momentum is due to some other physical process (such as AGN [11]) and not predominately due to mergers. 
These surprising results provide clues as to the formation history of Brightest Cluster Galaxies. It would be beneficial to have an increased sample of BCGs to add to our results, especially BCGs with companions, and more measurements of the companions to determine the probability of a bound BCG companion being a fast rotator. A larger sample size would also allow us to investigate the possible limit above which fast rotating galaxies do not exist. Our observations lead us to conclude that there is much diversity in the measurements of a BCG's angular momentum, dynamical mass, and merger history (Figure 2).

\section{References}

[1] Bournaud, F., Jog, C. J., \& Combes, F. 2007, A\&A, 476, 1179

[2] Jesseit, R., Cappellari, M., Naab, T., Emsellem, E., \& Burkert, A. 2009, MNRAS, 397, 1202

[3] Bois, M., Bournaud, F., Emsellem, E., et al. 2010, MNRAS, 406, 2405

[4] Bois, M., Emsellem, E., Bournaud, F., et al. 2011, MNRAS, 416, 1654

[5] Emsellem, E., Cappellari, M., Krajnović, D., et al. 2011, MNRAS, 414, 888

[6] Miller, C. J., Nichol, R. C., Reichart, D., et al. 2005, AJ, 130, 968

[7] Le F'evre, O., Saisse, M., Mancini, D., et al. 2003, in Society of Photo-Optical Instrumentation Engineers (SPIE) Conference Series, Vol. 4841, Society of Photo-Optical Instrumentation Engineers (SPIE) Conference Series, ed. M. Iye \& A. F. M. Moorwood, 1670-1681

[8] Emsellem, E., Cappellari, M., Krajnović, D., et al. 2007, MNRAS, 379, 401

[9] Cappellari, M., Scott, N., Alatalo, K., et al. 2013, MNRAS, 432, 1709

[10] Lotz, J. M., Jonsson, P., Cox, T. J., \& Primack, J. R. 2008, MNRAS, 391, 1137

[11] Martizzi, D., Teyssier, R., \& Moore, B. 2012, MNRAS, 420, 2859 